\title{
Anesthetics and human epidermal growth factor incorporated into anti-adhesive nanofibers provide sustained pain relief and promote healing of surgical wounds
}

This article was published in the following Dove Press journal:

International Journal of Nanomedicine

\author{
Ching-Wei Kao ${ }^{1,2}$ \\ Yuan-Yun Tseng ${ }^{3,4}$ \\ Kuo-Sheng Liu $^{5}$ \\ Yen-Wei Liu ${ }^{5}$ \\ Jin-Chung Chen ${ }^{6}$ \\ Hong-Lin $\mathrm{He}^{7}$ \\ Yi-Chuan $\mathrm{Kau}^{8}$ \\ Shih-Jung Liu ${ }^{2,9}$ \\ 'Department of Anesthesiology, Chiayi \\ Chang Gung Memorial Hospital, Chiayi, \\ Taiwan; ${ }^{2}$ Department of Mechanical \\ Engineering, Chang Gung University, \\ Taoyuan, Taiwan; ${ }^{3}$ Division of \\ Neurosurgery, Department of Surgery, \\ Shuang Ho Hospital, Taipei Medical \\ University, Taipei, Taiwan; ${ }^{4}$ Department \\ of Surgery, School of Medicine, College of \\ Medicine, Taipei Medical University, \\ Taipei, Taiwan; ${ }^{5}$ Department of Thoracic \\ and Cardiovascular Surgery, Linkou \\ Chang Gung Memorial Hospital, Taoyuan, \\ Taiwan; ${ }^{6} \mathrm{Graduate}$ Institute of Biomedical \\ Science, Chang Gung University, Taoyuan, \\ Taiwan; ${ }^{7}$ Department of Pathology, E-DA \\ Hospital, I-Shou University, Kaohsiung, \\ Taiwan; ${ }^{8}$ Department of Anesthesiology, \\ Linkou Chang Gung Memorial Hospital, \\ Taoyuan, Taiwan; ${ }^{9}$ Department of \\ Orthopedic Surgery, Linkou Chang Gung \\ Memorial Hospital, Taoyuan, Taiwan
}

Correspondence: Shih-Jung Liu Biomaterials Lab, Mechanical Engineering, Chang Gung University, 259, Wen-Hwa Ist Road, Kwei-Shan, Tao-Yuan 333, Taiwan

Tel +886321I 8166

Fax +88632118558

Email profsjliu5347@gmail.com
Background: This study exploited sheath-core-structured lidocaine/human EGF (hEGF)loaded anti-adhesive poly[(d,l)-lactide-co-glycolide] (PLGA) nanofibrous films for surgical wounds via a co-axial electrospinning technique.

Materials and methods: After spinning, the properties of the co-axially spun membranes were characterized by scanning electron microscopy, laser-scanning confocal microscopy, Fourier Transform Infrared spectrometry, water contact angle measurements, and tensile tests. Furthermore, a HPLC analysis and an ELISA evaluated the in vitro and in vivo release curves of lidocaine and hEGF from the films.

Results: PLGA anti-adhesion nanofibers eluted high levels of lidocaine and hEGF for over 32 and 27 days, respectively, in vitro. The in vivo evaluation of post-surgery recovery in a rat model demonstrated that no adhesion was noticed in tissues at 2 weeks after surgery illustrating the anti-adhesive performance of the sheath-core-structured nanofibers. Nanofibrous films effectively released lidocaine and hEGF for $>2$ weeks in vivo. In addition, rats implanted with the lidocaine/hEGF nanofibrous membranes exhibited greater activities than the control demonstrating the pain relief efficacy of the films.

Conclusion: The empirical outcomes suggested that the anti-adhesive nanofibrous films with extended release of lidocaine and hEGF offer post-operative pain relief and wound healing.

Keywords: sheath-core nanofiber, anti-adhesive membrane, sustained elution, lidocaine, hEGF

\section{Introduction}

Postsurgical adhesion formation is a significant clinical issue for most surgeons - it is a practically unavoidable outcome in abdominal and/or pelvic operations. Adhesions can incur major post-operation morbidity, bowel obstruction, infertility, and chronic pelvic pain or abdominal pain. ${ }^{1}$ The occurrence of postoperative intraabdominal adhesions accounts for $50-95 \%$ of females experiencing gynecological operation. ${ }^{2}$ Various treatments to forestall the formation or reformation of adhesions have been proposed.

Adhesion barriers are a promising therapy. They are implantable materials that decrease abnormal internal adhesions after operation by physically segregating the internal tissues and organs during healing. ${ }^{3}$ Adhesion barriers can be films, gels, or other materials that are employed between layers of tissue before the incision 
site is sutured. When implanted, the adhesion barrier acts as a physical shelter to segregate traumatized tissues to prevent coherence during healing. The barrier degrades and is resorbed by the body after the tissue surface cures (3-7 days).

Pain that arises after surgery is another important concern. Proper pain management-particularly postoperative pain management-is a primary apprehension for clinicians as well as for patients undergoing operation. ${ }^{4}$ Despite the advances in the field of pain management, some patients still cannot achieve complete ease from postoperative pain. Postoperative pain not only influences a patient's surgical outcome, welfare, and contentment from medical care, but also directly affects the evolution of tachycardia, hyperventilation, reduction in alveolar ventilation, transition to chronic pain, poor wound healing, and insomnia - this can improve surgical outcomes. ${ }^{5}$ The rapidly increasing number of complex surgical procedures that are performed in an outpatient setting has made perioperative and postoperative pain management very essential. ${ }^{6}$

Many different materials ${ }^{7-13}$ have been explored as potential anti-adhesive barriers - each with their own unique composition and limitations and advantages. The progress of drug/growth factor-embedded anti-adhesive barriers has been limited. Chen et al. ${ }^{14}$ proposed silver nanoparticles/ibuprofen-incorporated poly(L-lactide) fibrous films and evaluated their anti-infection/ anti-adhesion efficacies. Kao et al. ${ }^{15}$ developed lidocaine and ketorolac loaded poly[(d,1)-lactide-co-glycolide] (PLGA) anti-adhesive membranes to provide sustained release of analgesics for pain relief. An ideal barrier should possess the following characteristics: anti-adhesive, biocompatible, biodegradable, easily adherent to the injured surface, potent on the seeping surface, and able to relieve pain and promote healing. Unfortunately, such a barrier has not yet been described.

This study exploited lidocaine and human EGFincorporated PLGA nanofibers as an anti-adhesive membrane for surgical wound healing. Lidocaine hydrochloride is a local anesthetic, ${ }^{16}$ and $\mathrm{hEGF}^{17,18}$ promotes dermal wound curing via stimulation, proliferation, and migration of keratinocytes, endothelial cells, and fibroblasts; it facilitates dermal regeneration. ${ }^{19}$ After the electrospinning process, the properties of co-axially spun membranes were characterized. The in vitro/in vivo release patterns of the anesthetics and EGF from the fabricated nanofibrous films were explored. In addition, the biocompatibility and efficacy of the anesthetics/growth factor-embedded anti-adhesive films were assessed in rats receiving abdominal operations. Animal activities post-operation and histological analysis at the wound sites were also assessed.

\section{Materials and methods \\ Making of anesthetics/growth factor- eluting nanofibers}

Sheath-core-structured PLGA nanofibers were prepared using a co-axial electrospinning device built in our lab as shown in Figure 1. Adopted PLGA (50:50) possesses a molecular weight of $33 \mathrm{kDa}$ (Resomer RG 503; Sigma-Aldrich, St Louis, MO, USA). To prepare the anesthetics/growth factor loaded nanofibers, PLGA/lidocaine (720 mg: $180 \mathrm{mg}$ ) (Sigma-Aldrich), and hEGF (0.2 mg, hEGF-E9644; SigmaAldrich) were pre-mixed with $3 \mathrm{~mL}$ of hexafluoroisopropanol
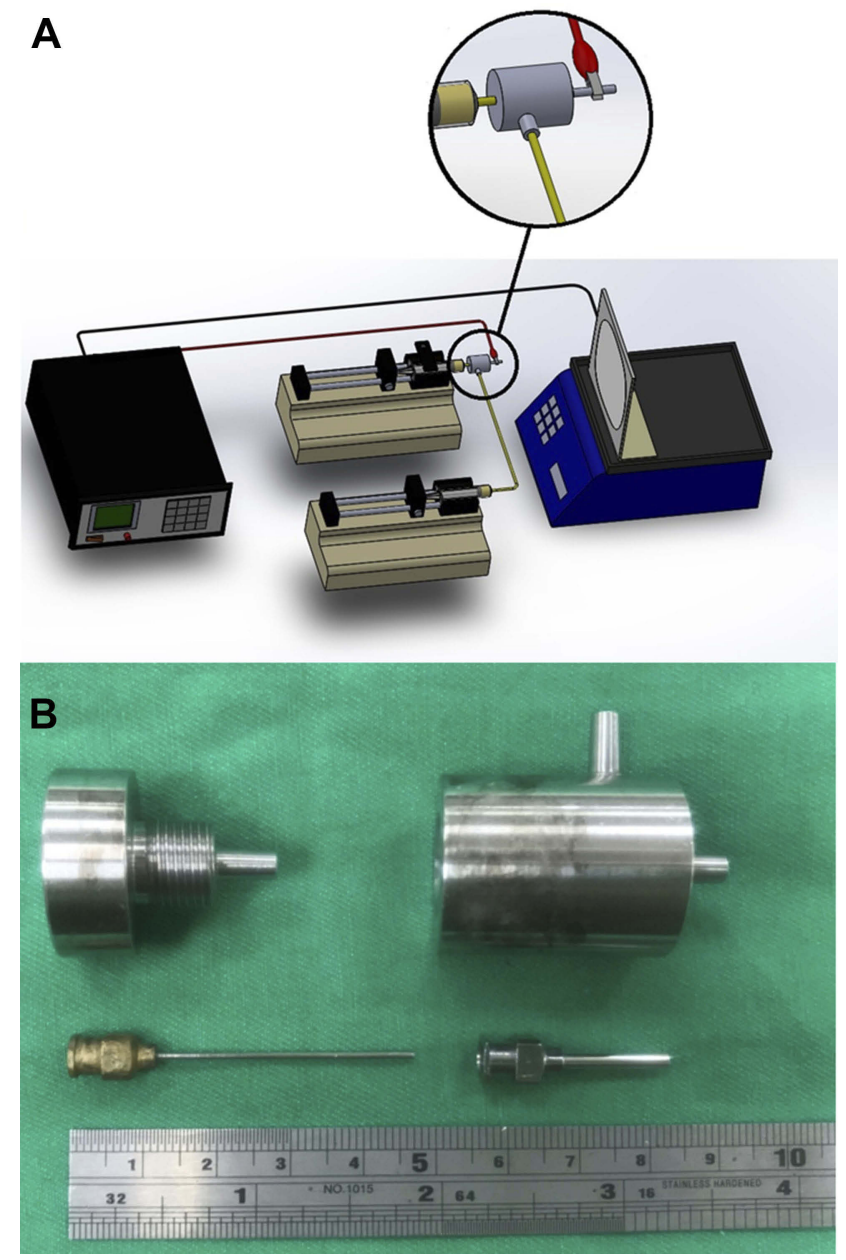

Figure I (A) Schematic of the experimental setup to co-axially electrospin the sheath-core-structured nanofibers; (B) photo of the co-axial nozzle and needles used for the experiments. 
(Sigma-Aldrich) and $1 \mathrm{~mL}$ of distilled water, respectively. The PLGA/lidocaine and hEGF solutions were then conveyed to the syringes for the sheath and the core layers, respectively, for co-axially electrospinning into nonwoven nanofibrous membranes. The delivery rate for the sheath layer was $0.6 \mathrm{~mL} / \mathrm{hr}$, and the transport rate for the core was $0.2 \mathrm{~mL} / \mathrm{hr}$. The voltage applied to the syringe tip was $8 \mathrm{kV}$, and the distance from the tip to the collection plates was $15 \mathrm{~cm}$.

\section{Evaluation of co-axially electrospun sheath-core films}

To evaluate the nanofiber size distribution, the morphology of co-axially spun nanofibers was observed on a scanning electron microscope (SEM) (JEOL Model JSM-7500F; JEOL, Tokyo, Japan) after they were coated with gold. The diameter distribution was acquired by analyzing SEM images of 50 randomly selected fibers for each test specimen $(N=3)$ utilizing a commercial Image $\mathrm{J}$ image software (National Institutes of Health, Bethesda, MD, USA).

The mechanical characteristics of the co-axially electrospun nanofibrous films were assessed by a Lloyd tensile tester (Ametek, USA). The sessile drop contact angle was measured on a contact angle goniometer (First Ten Angstroms, USA).

A laser-scanning confocal microscope (LSCM) (Leica TS SP8X, Japan) confirmed the existence of proteins in the co-axially electrospun nanofibers. PLGA was employed as the sheath layer while recombinant enhanced green fluorescent protein (reGFP) was utilized for the core. Co-electrospun nanofibers were observed at an excitation wavelength of $487 \mathrm{~nm}$.

To inspect the spectra of pure PLGA nanofibers and lidocaine/hEGF embedded PLGA nanofibers, the Fourier transform infrared (FTIR) analysis was completed on a Bruker Tensor 27 spectrophotometer at a resolution of $4 \mathrm{~cm}^{-1}$ and 32 scans. Nanofibrous film specimens were depressed as $\mathrm{KBr}$ discs and analyzed from 400 to $4,000 \mathrm{~cm}^{-1}$.

\section{In vitro release studies}

Nanofibrous specimens with an area of $1 \mathrm{~cm}$ by $1 \mathrm{~cm}$ were cut from the co-axially electrospun films and were put in test tubes $(N=3)$ containing $1 \mathrm{~mL}$ of buffered solution at $37^{\circ} \mathrm{C}$ for 1 day. The eluents were gathered and assayed. The fresh buffered solution was replenished daily, and the process was duplicated for 40 days.

The release pattern of lidocaine from the anesthetics/ growth factor-loaded nanofibers was evaluated by an HPLC assay. The mobile phase contained ammonium formate and methanol $(20 / 80[\mathrm{v} / \mathrm{v}])$. The absorbency wavelength was set at $254 \mathrm{~nm}$, and the flow rate was $1.0 \mathrm{~mL} / \mathrm{min}$. An hEGF ELISA kit (Cusabio Biotech., USA) measured hEGF in the eluent via absorbance at $450 \mathrm{~nm}$.

\section{Cell culture}

The cytotoxicity of anesthetics/hEGF-loaded PLGA nanofibers was examined by Cell Counting Kit-8 (CCK-8) assay (Sigma-Aldrich) of cell viability. Collected eluents at days 1-4 were put onto 96-well culture plates. Commercially available human foreskin fibroblasts (3T3, Food Industry Research and Development Institute, Hsinchu, Taiwan) were seeded $\left(1 \times 10^{4}\right.$ cells/well $)$ in Dulbecco's Modified Eagle's Medium at $37^{\circ} \mathrm{C}$ under the incubating condition that consists of $5 \% \mathrm{CO}_{2}$ and $95 \%$ ambient air for $48 \mathrm{hrs}$. Cell viability was monitored by CCK-8 assays and quantified using an ELISA reader.

\section{In vivo animal tests}

The study in vivo with animal used a rat incisional pain model. ${ }^{15}$ All animal-related processes gained approval from Institutional Animal Care and Use Committee of Chang Gung University. All of the studied animals were cared for in a manner consistent with the regulations of the Ministry of Health and Welfare, Taiwan under the supervision of a licensed veterinarian. Forty adult Wistar rats ( $\sim 250 \mathrm{~g}$ each) were enrolled for the in vivo examinations: 20 rats were administered for the in vivo anesthetics/growth factor level studies, and the other 20 rats were used for the animal activity tests. For the anesthetics/growth factor level experiments, the rats were anesthetized via isoflurane.

A 4-cm incision was placed in the lower abdomen of each animal. After the implantation of the lidocaine/hEGF nanofibrous films $(40 \mathrm{~mm} \times 20 \mathrm{~mm}$ in size $)$, the wound was closed with 3-0 Vicryl sutures. The 20 animals were sacrificed for local tissue sampling at post-operative days 1, 3, 7, and 14 (five animals at each time point, $N=5$ ) via anesthesia overdose. The blood samples were collected via cardiac puncture. Lidocaine and hEGF concentrations in the specimens were measured with an HPLC assay and an ELISA kit, respectively. Tissue from the wounds treated with the drug/growth factor-loaded nanofibers was sampled for histological examination of epithelialization and granulation.

\section{Post-surgery activity}

A second cohort of 20 rats was partitioned into four groups (five animals per group, $N=5$ ) for activity testing. Group A 
had no surgery as a control. Group B had the operation only, Group C had surgery plus pure PLGA nanofibers, Group D received surgery and lidocaine/hEGF-loaded PLGA nanofibers. The post-surgery activity of each animal was assessed utilizing a $50 \mathrm{~cm} \times 50 \mathrm{~cm} \times 50 \mathrm{~cm}$ cage fitted with nine diffusion-scan type sensors. ${ }^{15}$ The sensors detected the movements of animal in the cage. As the animal migrated, the sensor in the "arriving" area was triggered. A micro-processing unit equipped with an acquisition interface monitored the total movement counts. The activity of every animal was inspected and recorded over 7 days.

\section{Statistical analysis}

A least significance difference evaluated the between-group differences for continuous variables, and the Chi-squared test was employed to assess the activity performance. Acquired data were analyzed utilizing SPSS software (Version 12.0; SPSS Inc., Chicago, IL, USA). Probabilities of 0.05 or less were considered to be significant.

\section{Results}

\section{Evaluation of anesthetics/growth factor embedded nanofibers}

Lidocaine/growth factor-embedded nanofibrous films were satisfactorily fabricated using a co-axial spinning process. Figure 2 shows the SEM images of the spun fibers and fiber size distributions. The estimated diameters were $780.0 \pm 552.1 \mathrm{~nm}$. LSCM examined the distribution of protein in the co-axially spun nanofibers. Figure 3 displays the image of string-like green signals of reGFP demonstrating the protein's bioactivity in the sheath-core-structured nanofibers after co-axial electrospinning. A homogeneous protein distribution was confirmed in the nanofibers.

An FTIR spectrometer simultaneously collects highspectral-resolution data over a wide spectral range. It is a technique widely employed to obtain the infrared spectrum of absorption or emission of a solid, liquid or gas. Figure 4 shows the FTIR spectra of pure PLGA films and lidocaine/hEGF-loaded PLGA films. A new vibration peak at $3,400 \mathrm{~cm}^{-1}$ was detected due to the $\mathrm{N}-\mathrm{H}$ bonds of lidocaine. ${ }^{20}$ The peak at $1,725 \mathrm{~cm}^{-1}$ $(\mathrm{C}=\mathrm{O}$ bond) was promoted with the incorporation of anesthetics. In addition, the vibration near $1,300 \mathrm{~cm}^{-1}$ might be attributable to the $\mathrm{C}-\mathrm{O}$ bond increase in lidocaine. The FTIR spectra assay confirmed that the lidocaine was sufficiently embedded in the PLGA film.
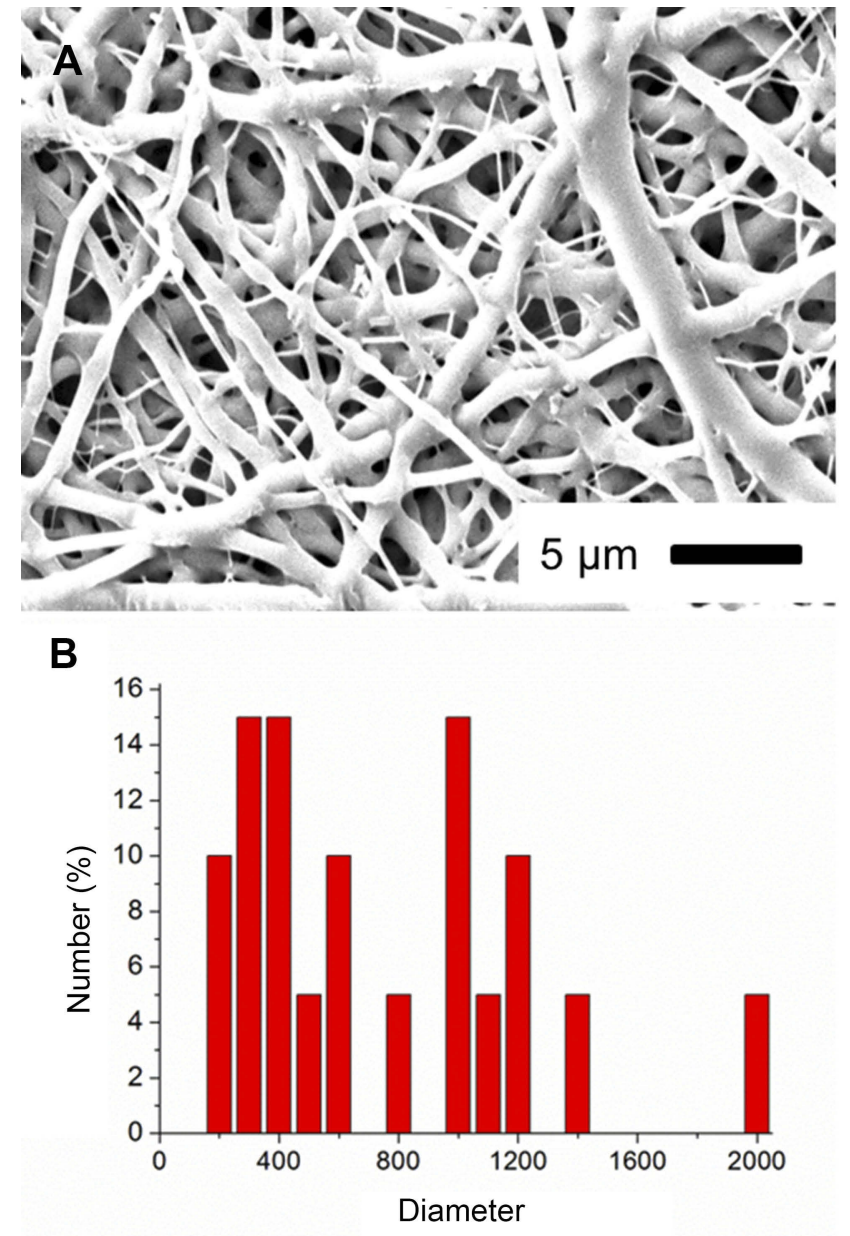

Figure 2 SEM image and fiber size distribution of lidocaine/hEGF-loaded nanofibers. Abbreviations: SEM, scanning electron microscope; hEGF, human epidermal growth factor.

Figure 5 shows that the water contact angles of pure PLGA, lidocaine loaded PLGA, and lidocaine/hEGFloaded PLGA nanofibers were 127.8, 52.3, and 60.1, respectively. The anesthetics made the PLGA nanofibrous films more hydrophilic.

The tensile test results in Figure 6 suggest that the ultimate strength (maximum strain) of the pure PLGA, lidocaine loaded PLGA, and lidocaine/hEGF embedded nanofibrous films were 4.62 MPa (140.7\%), 2.7 MPa (57.2\%), and $2.33 \mathrm{MPa}(23.3 \%)$, respectively. The tensile strength decreased upon addition of lidocaine and hEGF.

\section{Release patterns of lidocaine and hEGF from the nanofibers}

Figure 7 shows the daily and accumulated in vitro release patterns of lidocaine from nanofibrous films. The anesthetics displayed a triphasic release behavior, ie, a burst elution at 1-2 days, a steady and gradually diminishing drug elution at days $3-10$, and a second peak release at days 12-13 after which the 


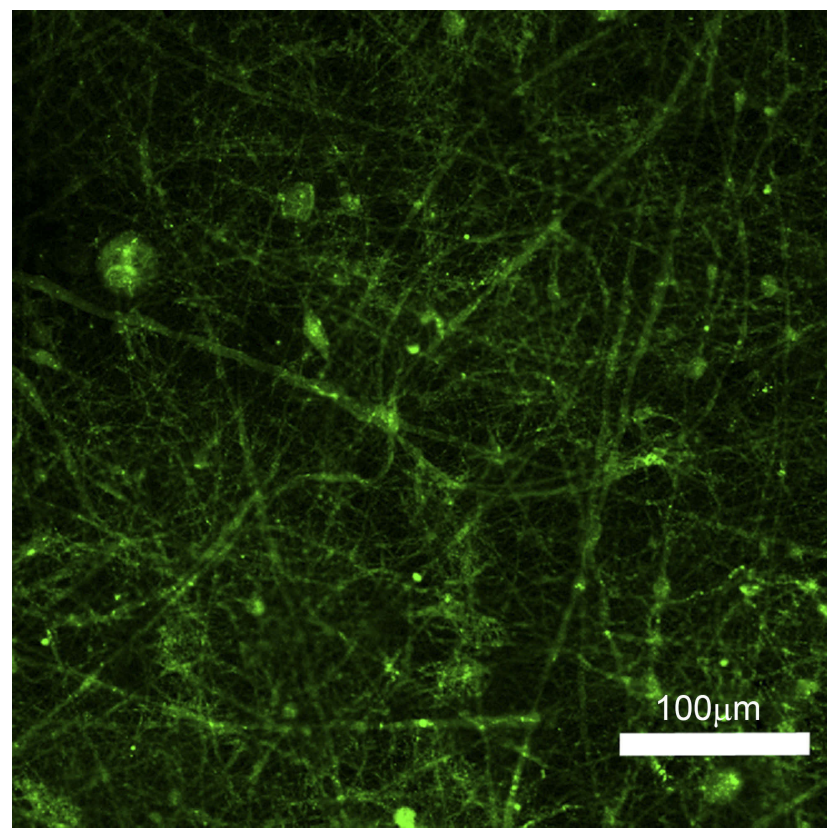

Figure 3 Laser scanning confocal microscopy images of reGFP in co-axial electrospun nanofibers.

Abbreviation: reGFP, recombinant enhanced green fluorescent protein.

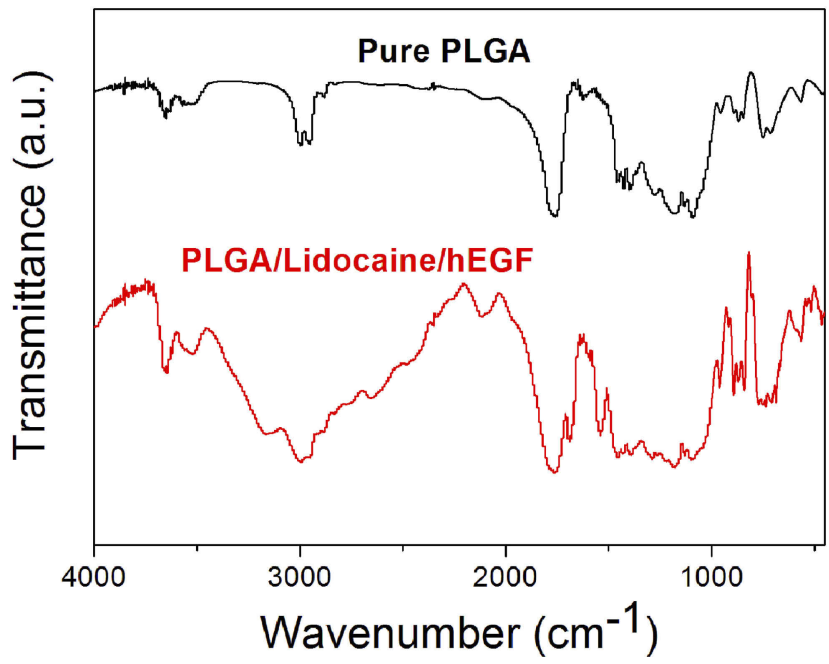

Figure 4 FTIR spectra of electrospun pure PLGA and lidocaine/hEGF-loaded PLGA nanofibers.

Abbreviations: FTIR- fourier-transform infrared spectroscopy; hEGF, human epidermal growth factor; PLGA, poly[(d,I)-lactide-co-glycolide]; FTIR, fourier-transform infrared spectroscopy.

release of drugs diminished. The curve has only a tiny SD indicating that the incorporated anesthetics were evenly allocated in the PLGA nanofibers. In addition, the electrospun nanofibrous films liberated lidocaine for over 30 days in vitro. Figure 8 displays the in vivo release curves of lidocaine in tissue and blood. The levels of anesthetics remained high at the target area for over 2 weeks, but they were low systemically.

Figure 9 shows that the sheath-core-structured nanofibers provide sustained release of high levels of hEGF for

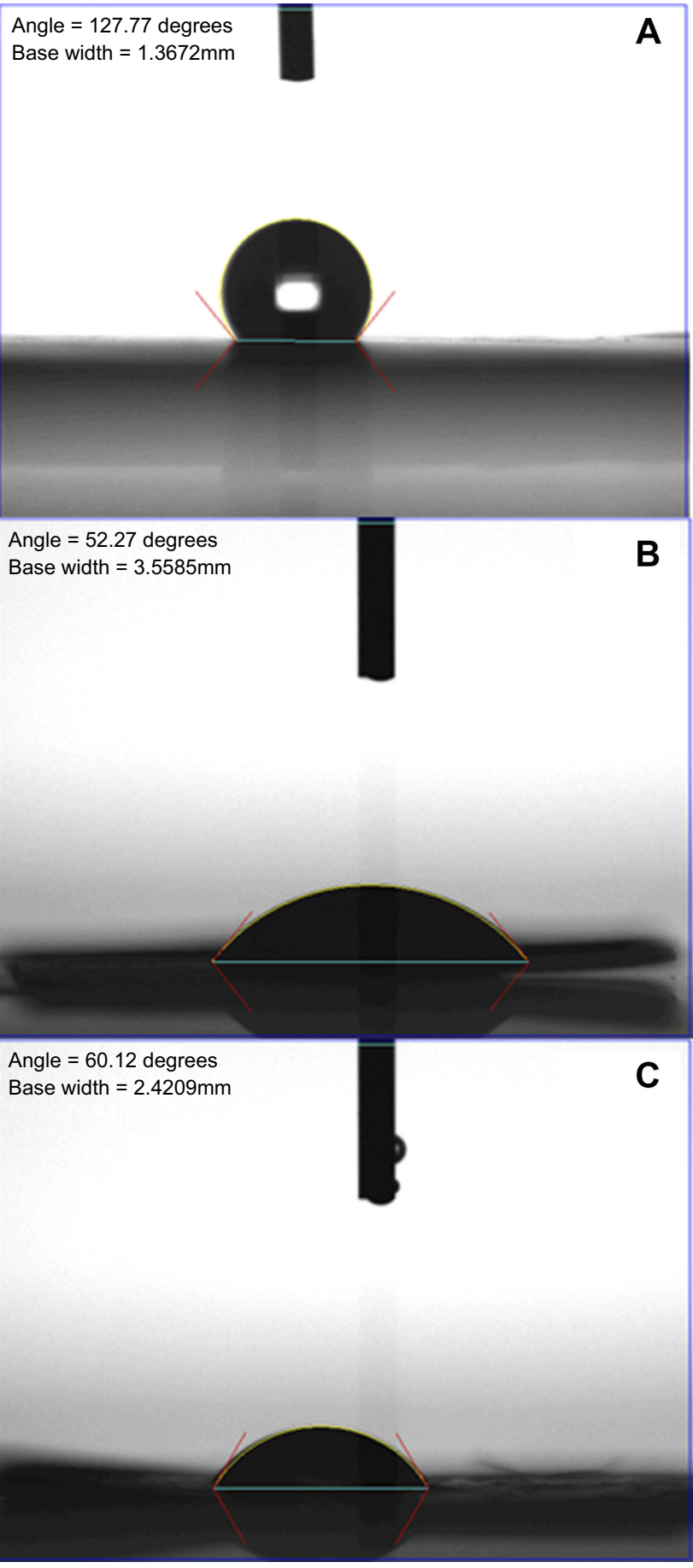

Figure 5 Measured contact angles. (A) Pure PLGA nanofibers, 127.8, (B) lidocaineincorporated nanofibers, 52.3, and (C) lidocaine/hEGF-loaded nanofibers, 60.I. Abbreviations: hEGF, human epidermal growth factor; PLGA, poly[(d,I)-lactide-coglycolide].

over than 27 days. Meanwhile, the result in Figure 10 indicates that the nanofibrous films release high levels of hEGF in vivo for over 14 days. The effectiveness of eluted hEGF was determined by the cell viability assay. The results in Figure 11 illustrated that all nanofibers show 


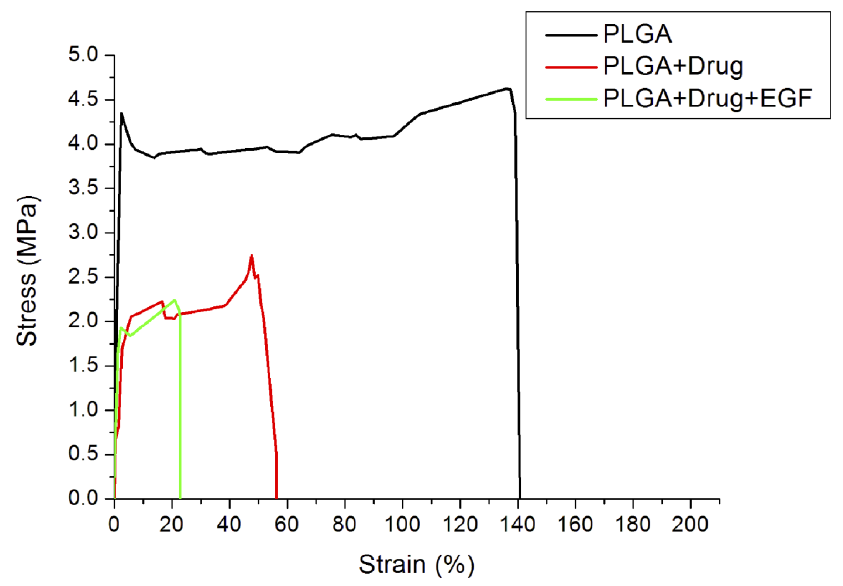

Figure 6 Stress-strain curve of pure PLGA, lidocaine-incorporated, and lidocaine/ hEGF-loaded sheath-core-structured nanofibers.

Abbreviations: hEGF, human epidermal growth factor; PLGA, poly[(d,I)-lactideco-glycolide].
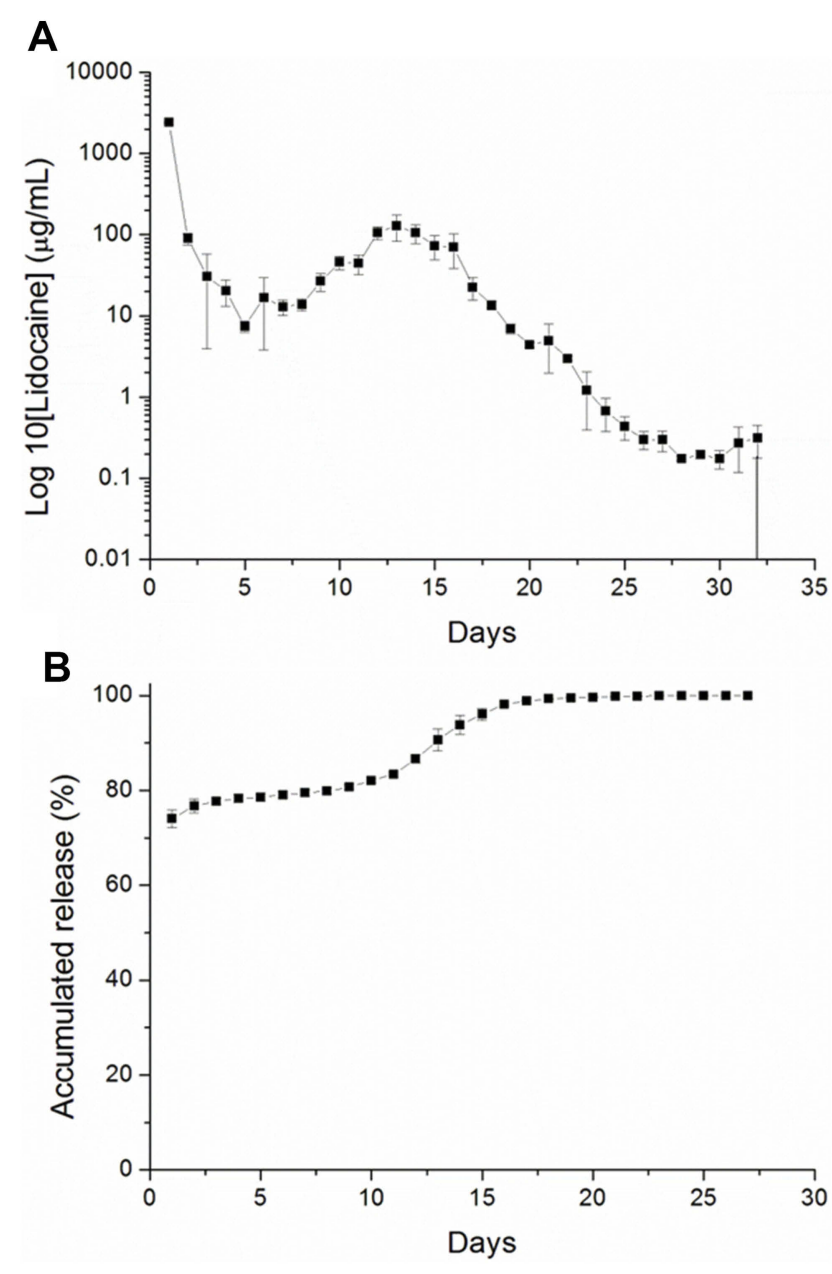

Figure 7 In vitro release patterns of anesthetics from the nanofibrous films. Notes: (A) Daily release, (B) accumulated release.

no signs of cytotoxicity. In addition, the hEGF promoted the proliferation of human fibroblasts during the first 3 days demonstrating that hEGF was not deactivated during electrospinning.

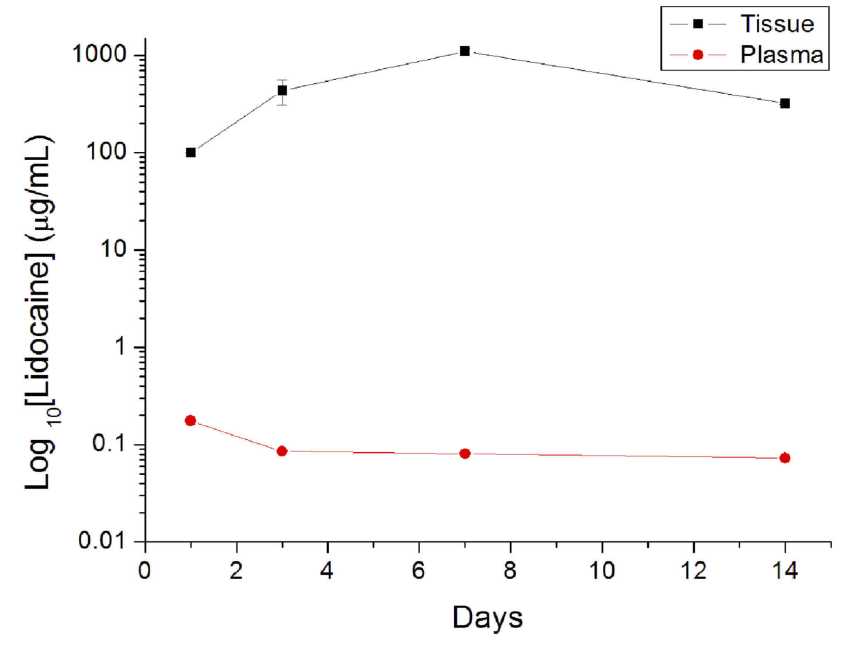

Figure 8 In vivo release of lidocaine from the nanofibrous films.

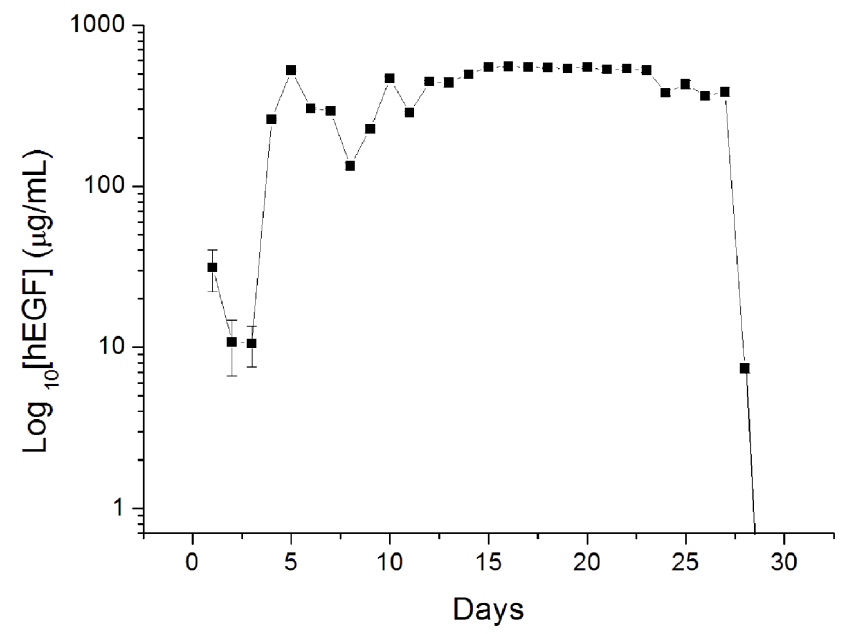

Figure 9 In vitro elution profiles of hEGF from the nanofibrous films. Abbreviation: hEGF, human epidermal growth factor.

\section{Efficacy of liberated anesthetics}

The total counts of animal activities for the four groups over the seven post-operative days are displayed in Figure 12. The number of triggered counts in group D (surgery followed by the deployment of lidocaine/hEGF loaded PLGA membrane) was significantly greater than that in group $\mathrm{B}$ (surgery only) at Sensor No. $1(P<0.05)$ and Sensor No. $3(P<0.01)$ despite the fact that the recorded total counts did not show significant activity level differences among the various groups $(P>0.05)$.

\section{Post-surgery anti-adhesion and histological examination}

The films in rats could not be observed at 2 weeks postimplantation. No tissue adhesion was noted (Figure 13) 


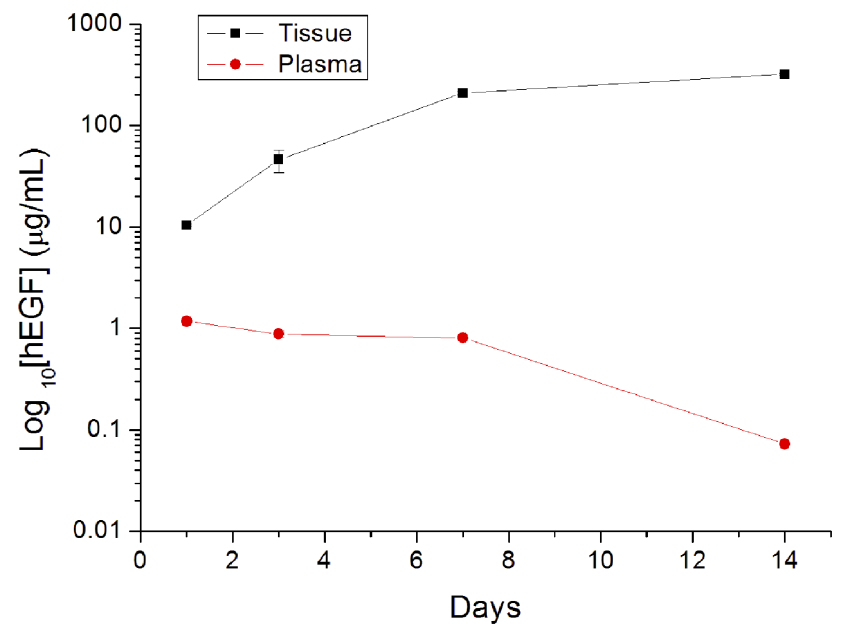

Figure 10 In vivo release of hEGF from the nanofibrous films. Abbreviation: hEGF, human epidermal growth factor.

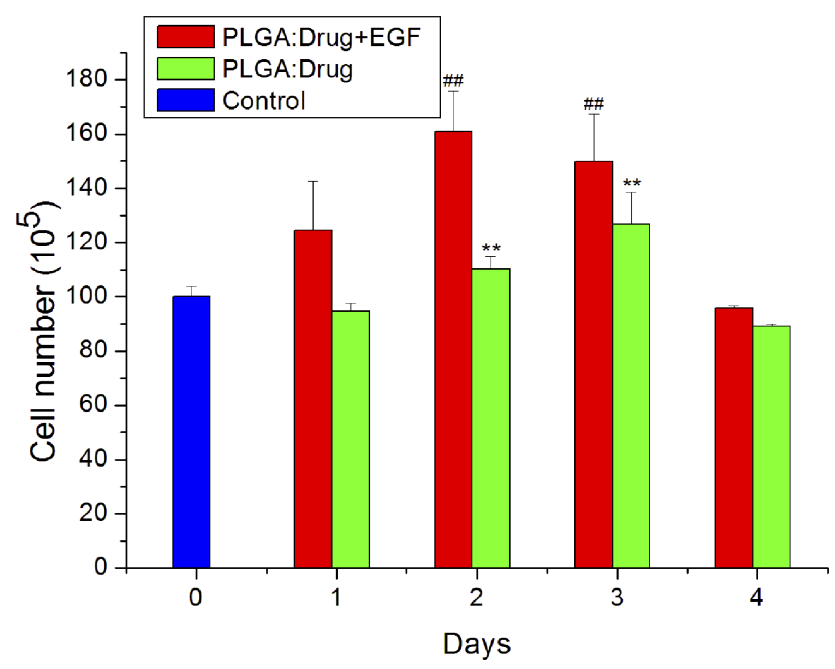

Figure I I Cell viability of electrospun lidocaine/hEGF nanofibers ( $\left.{ }^{\#} P<0.01, * * P<0.01\right)$. Abbreviations: hEGF, human epidermal growth factor; EGF, epidermal growth factor.

confirming the anti-adhesive capacity of the anesthetics/ growth factor-loaded nanofiber film.

Figure 14 shows the hematoxylin \& eosin (H\&E) stained sections at wounds implanted with drug-eluting membranes. A foreign body reaction was noted at day 1 and was characterized by disruption of epidermal and dermal layers with mixed infiltrates of neutrophils and lymphocytes. On day 3, fibroblastic proliferation and mixed infiltrates of lymphocytes and plasma cells were observed in the subcutis and muscular layer.

\section{Discussion}

This study developed lidocaine/hEGF-incorporated sheathcore-structured anti-adhesive nanofibrous PLGA membranes. PLGA is an extensively explored biodegradable polymers that is approved for use in drug formulations and medical devices by the Food and Drug Administration. It is versatile in terms of molecular weights, compositions, and degradation rates for drug delivery. ${ }^{21,22}$ PLGA has excellent biocompatibility, a long-standing track record, and is suitable for continuous drug release; thus, it has been widely used in biomedicine.

Co-axial electrospinning is a cost-effective and efficient technique for preparing core-sheath-structured nanofibers. Spun nanofibers with pharmaceuticals encapsulated in the core can offer controlled yet sustained release. Furthermore, their possessed high surface area and three-dimensional nanofiber networks permit the electrospun fibers to mimic native extracellular matrices. These features of the nanofibers give them great potential in applications such as drug delivery and tissue engineering. Proteins, growth factors, antibiotics, and many other agents can be successfully embedded into coaxial fibers for drug delivery. The biggest advantage of the core-sheath design is that these agents remain bioactive after the electrospinning process due to the protection of the sheath layer.

Surgical pain is a disagreeable sensation that originates from a physical intervention. The pain is from the incision, the procedure itself, the closing of the wound, and any force that is applied during the procedure. Pain control following surgery is a priority for both the patients and doctors. Inadequately administered post-surgery pain can elicit complications and prolonged rehabilitation. Unconstrained acute pain can result in the development of chronic pain with compromised life quality. Proper pain relief gives rise to curtailed hospital stays, subsided treatment costs, and improved patient satisfaction. Furthermore, good pain control helps accelerate the healing and lessen the risk of evolving some post-operative complications such as pneumonia and blood clots. Patients may receive a nerve block to manage the pain after treatment. While an epidural governs pain over a broad region of the body, a nerve block only manages pain within a smaller area of the body. The main advantage of a nerve block is that it uses smaller amounts of narcotic medication. This will lead to fewer side effects such as nausea, vomiting, itching, and drowsiness. The nanofibrous films developed here could provide an extended release of anesthetics at the wound site as a topical anesthetic patch; they facilitated prolonged relief of postoperative pain.

Wound healing is a complex and dynamic process of replacing devitalized and missing cellular structures and 


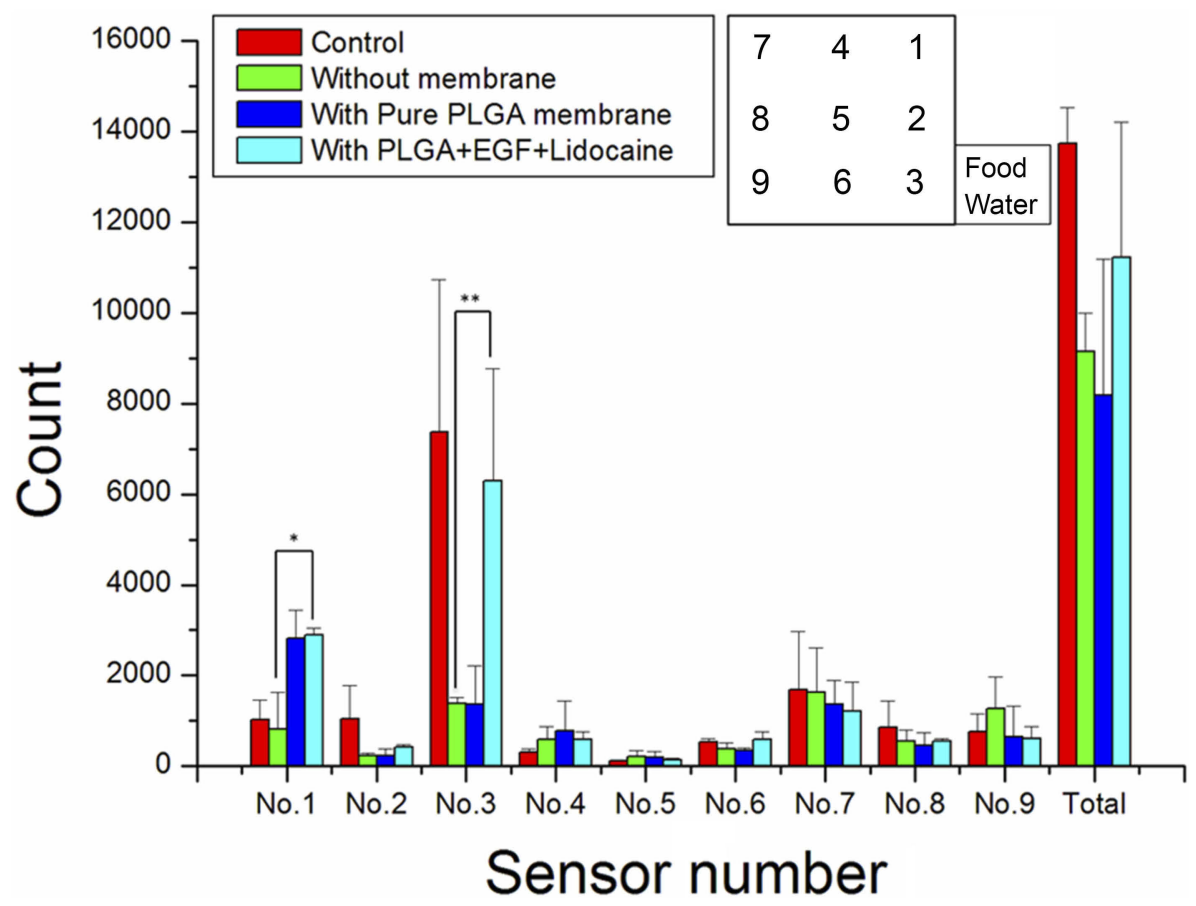

Figure 12 Activities in the rats.

Abbreviation: EGF, epidermal growth factor; PLGA, poly[(d,I)-lactide-co-glycolide].

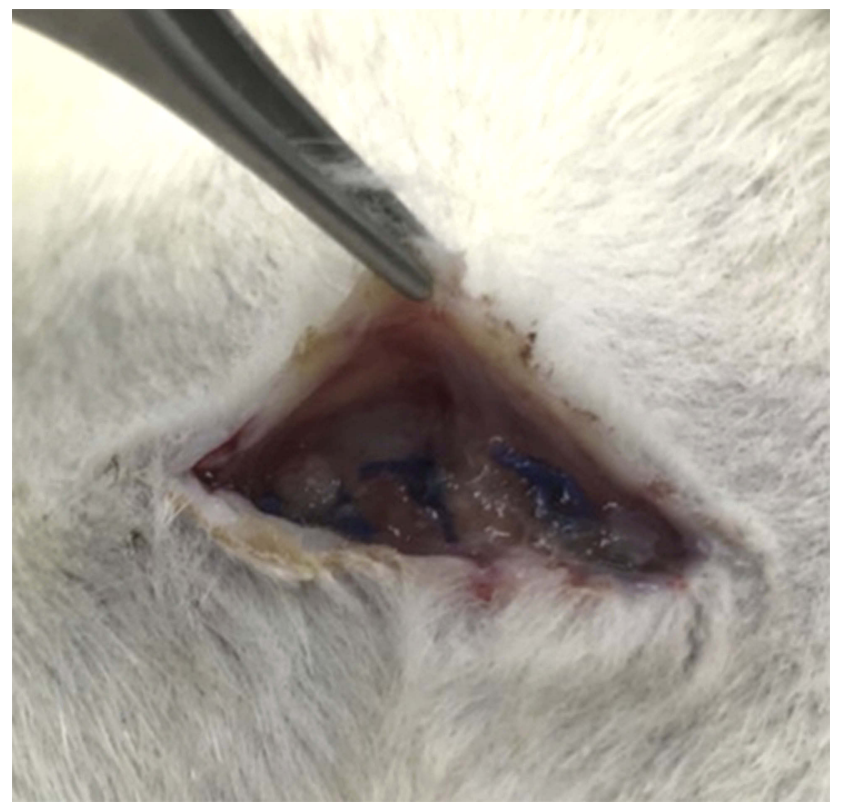

Figure 13 No tissue adhesion was observed for the wounds implanted with the sheath-core membranes.

tissue layers. It is a series of biologic events that begin as hemostasis but then comprise an inflammatory response. This includes the formation of connective tissue, the covering of the wound with epithelium, and wound remodeling. Efficient wound healing relies on growth factor and cytokine signals that enhance keratinocyte dedifferentiation and migration for re-epithelialization. ${ }^{18}$ EGF takes part in dermal wound healing via stimulation, proliferation, and migration of keratinocytes, endothelial cells, and fibroblasts; it facilitates dermal regeneration. EGF is a dominant signaling molecules that stimulates epithelial cell motility for reepithelialization. ${ }^{19}$ It also is a major stimulator of fibroblast migration and wound contraction. The hEGF-loaded nanofibrous membranes could act as ideal EGF-eluting scaffolds for promoting surgical wounds.

Drug release from biodegradable vehicles can be generally composed of three stages: a burst, a diffusion-dominated stage, and a degradation-governed stage. During coaxial electrospinning, most pharmaceuticals are distributed in the volume of the PLGA mat. Nevertheless, a few drugs may be dispersed on the surfaces of the nanofibers thus leading to the initial burst. After the burst, the drugrelease curve is both governed by the diffusion effect and the polymer degradation. A comparatively constant decline in the release of lidocaine was thus noticed. A gentler and steadier elution of hEGF at the core was also observed due to the shelter of the sheath layer. The outcomes confirm that the co-axially spun PLGA drug-eluting films liberate high levels of lidocaine and hEGF for over 27 and 14 days, respectively, which is advantageous for pain control and wound healing. 

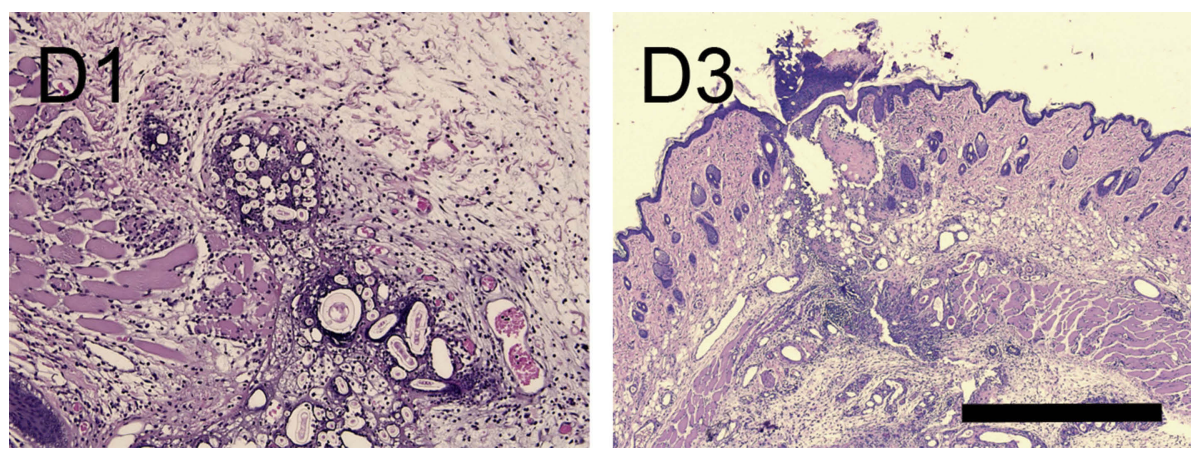

Figure I 4 Wound healing in hEGF/lidocaine-incorporated nanofibers at post-surgical days I and 3 (scale bar: I mm). A foreign body reaction was noted at day I (DI), while fibroblastic proliferation and mixed infiltrates of lymphocytes and plasma cells were observed in the subcutis and muscular layer on day 3 (D3). Abbreviation: hEGF, human epidermal growth factor.

Our previous work has demonstrated that both lidocaine and ketorolac-incorporated nanofibers can offer extended pain relief for surgical wounds. ${ }^{15}$ One major limitation of local anesthetics is systemic blood toxicity. The results illustrate that the concentration of lidocaine remains low in the plasma and is much lower than that in the local area. This offers high concentrations at the target area with minimal systemic side effects. While rats given lidocaine/ketorolac nanofibers exhibited effective pain relief, ${ }^{15}$ the rats implanted with lidocaine-only nanofibers here had no significant pain relief compared to the control. This suggests that multiple-analgesics should be used for the preparation of anti-adhesive membranes for sustained relief of surgical pain.

Despite these preliminary findings, there are limitations associated with this study. The first is the comparatively low number of animals used. The second limitation is that the relationship of the discoveries in this work to humans with operational pain remains unanswered. These will be the topic of future studies.

\section{Conclusion}

In this study, we exploited biodegradable sheath-corestructured anesthetics and growth factor-loaded nanofibers and evaluated their effectiveness for pain relief and healing promotion. These results suggest that the coaxially electrospun nanofibers can offer adequately sustainable lidocaine and hEGF at the target site for over 2 weeks post-operation. The nanofibrous films also have anti-adhesion features. Nanofibers with sustainable anesthesia and growth factor release can be used as anti-adhesion films. This would lead to extensive pain relief and accelerated wound healing.

\section{Acknowledgments}

This work was financially sponsored by the Ministry of Science and Technology, Taiwan (Contract No. 1072221-E-182-017) and the Chang Gung Memorial Hospital (Contract No. CMRPD2H0032).

\section{Disclosure}

The authors report no conflicts of interest in this work.

\section{References}

1. Schnuriger B, Barmparas G, Branco BC, Lustenberfer T, Inaba K, Demetriades D. Prevention of postoperative peritoneal adhesions: a review of the literature. Amer J Surg. 2011;201(1):111-121. doi:10.1016/j.amjsurg.2010.02.008

2. Hellebrekers BWJ, Trimbos-Kemper GCM, van Blitterswijk CA, Bakkum EA, Trimbos JBMZ. Effects of five different barrier materials on postsurgical adhesion formation in the rat. Hum Reprod. 2000;15(6):1358-1363.

3. Ntourakis D, Katsimpoulas M, Tanoglidi A, et al. Adhesions and healing of intestinal anastomoses: the effect of anti-adhesion barriers. Surg Innov. 2016;23(3):266-276. doi:10.1177/1553350615610653

4. Neil MJE, Macrae WA. Post surgical pain - the transition from acute to chronic pain. Rev Pain. 2009;3(2):6-9. doi:10.1177/ 204946370900300203

5. Shoar S, Esmaeili S, Safari S. Pain management after surgery: a brief review. Anesth Pain Med. 2012;1(3):184-186. doi:10.5812/ kowsar.22287523.3443

6. Garimella V, Cellini C. Postoperative pain control. Clin Colon Rectal Surg. 2013;26(3):191-196. doi:10.1055/s-0033-1351138

7. Niwa D, Koide M, Fujie T, Goda N, Takeoka S. Application of nanosheets as an anti-adhesion barrier in partial hepatectomy. J Biomed Mater Res B. 2013;101(7):1151-1258. doi:10.1002/jbm.b.32937

8. Bae SH, Son SR, Kumar Sakar S, et al. Evaluation of the potential anti-adhesion effect of the PVA/Gelatin membrane. $J$ Biomed Mater Res B. 2014;102(4):840-849. doi:10.1002/jbm.b.33066

9. Lo H-Y, Kuo H-T, Huang Y-Y. Application of polycaprolactone as an anti-adhesion biomaterials film. Artif Organs. 2010;34(8):648-653. doi:10.1111/j.1525-1594.2009.00949.x

10. Lee M-W, Tsai H-F, Wen S-M, Huang C-H. Photocrosslinkable gellan gum film as an anti-adhesion barrier. Carbohydr Polym. 2012;90:1132-1138. doi:10.1016/j.carbpol.2012.06.064 
11. Liu S, Zhao J, Ruan H, et al. Antibacterial and anti-adhesion effects of the silver nanoparticles-loaded poly(L-lactide) fibrous membrane. Mater Sci Eng C. 2013;33:1176-1182. doi:10.1016/j.msec.2012.12.008

12. Lim JI, Kang MJ, Lee W-K. Lotus-leaf-like structured chitosanpolyvinyl pyrrolidone films as an anti-adhesion barrier. Appl Surf Sci. 2014;320:614-619. doi:10.1016/j.apsusc.2014.09.087

13. Sotiri I, Overton JC, Waterhouse A, Howell C. Immobilized liquid layers: a new approach to anti-adhesion surfaces for medical applications. Exp Biol Med. 2016;241:909-918. doi:10.1177/1535370216640942

14. Chen S, Wang G, Wu T, et al. Silver nanoparticles/ibuprofen-loaded poly (L-lactide) fibrous membrane: anti-infection and anti-adhesion effects. Int J Mol Sci. 2014;15(8):14014-14025. doi:10.3390/ijms150814014

15. Kao CW, Lee D, Wu MH, Chen JK, He HL, Liu SJ. Lidocaine/ ketorolac-loaded biodegradable nanofibrous anti-adhesive membranes that offer sustained pain relief for surgical wounds. Inter $J$ Nanomed. 2017;12:5893-5901. doi:10.2147/IJN.S140825

16. Weibel S, Jokinen J, Pace NL, et al. Efficacy and safety of intravenous lidocaine for postoperative analgesia and recovery after surgery: a systematic review with trial sequential analysis. Brit $J$ Anaes. 2016;6:770-783. doi:10.1093/bja/aew101
17. Steed DL. The role of growth factors in wound healing. Surg Clin North Am. 1997;77(3):575-586.

18. Bodnar RJ. Epidermal growth factor and epidermal growth factor receptor: the Yin and Yang in the treatment of cutaneous wounds and cancer. Adv Wound Care. 2013;2(1):24-29. doi:10.1089/wound.2011.0326

19. Gonzalez ACDO, Costa TF, Andrade ZDA, Medrado ARAP. Wound healing - a literature review. An Bras Dermatol. 2016;91(5):614-620. doi:10.1590/abd1806-4841.20164741

20. Trovatti E, Silva NHCS, Duarte IF, et al. Biocellulose membranes as supports for dermal release of lidocaine. Biomacromolecules. 2011;12(11):4162-4168. doi:10.1021/bm201303r

21. Xu YH, Kim CS, Saylor DM, Koo D. Polymer degradation and drug delivery in PLGA-based drug-polymer applications: a review of experiments and theories. J Biomed Mater Res B. 2017;105 (6):1692-1716. doi:10.1002/jbm.b.33648

22. Houchin ML, Topp EM. Chemical degradation of peptides and proteins in PLGA: a review of reactions and mechanisms. J Pharm Sci. 2008;97(7):2395-2404. doi:10.1002/jps.21176
International Journal of Nanomedicine

\section{Publish your work in this journal}

The International Journal of Nanomedicine is an international, peerreviewed journal focusing on the application of nanotechnology in diagnostics, therapeutics, and drug delivery systems throughout the biomedical field. This journal is indexed on PubMed Central, MedLine, CAS, SciSearch ${ }^{\mathbb{B}}$, Current Contents ${ }^{\mathbb{R}} /$ Clinical Medicine,

\section{Dovepress}

Journal Citation Reports/Science Edition, EMBase, Scopus and the Elsevier Bibliographic databases. The manuscript management system is completely online and includes a very quick and fair peer-review system, which is all easy to use. Visit http://www.dovepress.com/ testimonials.php to read real quotes from published authors. 